\title{
O.S.P.
}

L'orientation scolaire et professionnelle

$29 / 2 \mid 2000$

Diplôme et marché du travail

\section{Dans les premières années de vie active}

Diploma and labour market: introduction

Jean-François Giret

\section{(2) OpenEdition}

Journals

Édition électronique

URL : http://journals.openedition.org/osp/5902

DOI : 10.4000/osp.5902

ISSN : 2104-3795

Éditeur

Institut national d'étude du travail et d'orientation professionnelle (INETOP)

Édition imprimée

Date de publication : 15 juin 2000

ISSN : 0249-6739

Référence électronique

Jean-François Giret, « Dans les premières années de vie active », L'orientation scolaire et professionnelle [En ligne], 29/2 | 2000, mis en ligne le 01 juin 2018, consulté le 16 décembre 2020. URL : http:// journals.openedition.org/osp/5902 ; DOI : https://doi.org/10.4000/osp.5902

Ce document a été généré automatiquement le 16 décembre 2020.

(c) Tous droits réservés 


\title{
Dans les premières années de vie active
}

\author{
Diploma and labour market: introduction
}

Jean-François Giret

\section{Introduction}

Dans un contexte d'allongement sans précédent de la formation initiale, la question de l'insertion des jeunes diplômés est un phénomène social particulièrement sensible et un enjeu majeur pour la recherche. Paradoxalement, depuis plusieurs années, des générations de plus en plus diplômées s'insèrent de plus en plus mal, même si la reprise économique de 1997 semble s'être traduite par une " embellie » des conditions d'insertion (Minni \& Poulet, 1999a, 1999b).

2 Le passage à l'enseignement supérieur de masse et l'influence des politiques éducatives ont largement influencé les trajectoires scolaires et universitaires sans pour autant réduire la période de transition entre les études et l'emploi. L'analyse des parcours d'insertion professionnelle ne fait plus ressortir un schéma unique d'entrée dans la vie active. Les parcours d'insertion, parfois plus longs, parfois plus précaires, sont souvent caractérisés par de fortes disparités au sein de mêmes cohortes et de mêmes niveaux de formation. Si l'observation des situations individuelles, mois par mois, montre que cette diversité se réduit progressivement dans le temps, elle reste très importante en début de vie active. Les jeunes mettent plusieurs années pour accéder à une position stabilisée sur le marché du travail dans des emplois souvent moins qualifiés que leurs aînés. La littérature empirique sur l'insertion des jeunes montre que plusieurs facteurs expliquent cette diversité des trajectoires d'entrée dans la vie active. Cet article se propose d'étudier plus particulièrement le rôle du diplôme et des filières de formation. Il est évident que le marché du travail des jeunes n'est pas un marché complètement cloisonné et que les jeunes entrent en concurrence avec d'autres générations souvent moins diplômées mais plus expérimentées (Béduwé \& Espinasse, 1996). Analyser l'influence du diplôme au sein d'une génération de jeunes ne restitue donc qu'une 
vision partielle de la concurrence sur le marché du travail. Il permet cependant de comprendre comment sont évalués les diplômes par les employeurs au sein d'une population plus ou moins homogène, et donc de fournir une évaluation à l'augmentation de la demande sociale d'éducation (D'Iribarne, 1986; Mansuy \& Méhaut, 1999). Si l'on considère la période d'insertion comme une file d'attente pour accéder à des positions stabilisées dans l'emploi, on peut se demander pourquoi certains jeunes diplômés se placent d'emblée en haut de cette file d'attente et même parfois l'évitent, alors que d'autres sont relégués au bas de cette file. Comme le propose Vincens (1999), il est possible d'introduire le concept "d'avantage relatif » : le diplôme de certaines filières confère un avantage relatif pour l'accès à certaines catégories d'emploi. Autrement dit, pour ce type d'emplois, la formation initiale fournit une productivité supérieure aux jeunes diplômés de ces filières. Il ne s'agit pas d'un avantage absolu, sauf pour quelques professions réglementées, mais d'un avantage relatif, qui dépendra à la fois des caractéristiques de la filière de formation, et des caractéristiques individuelles des jeunes.

Une analyse longitudinale de l'insertion, comme nous la proposons dans cet article, permet de tester la réalité de cet avantage relatif entre des jeunes issus d'une même cohorte. D'un point de vue théorique, nous examinerons plus précisément les causes de cet avantage conféré par le diplôme sur le marché du travail des jeunes à partir d'une approche principalement économique. Ce sera l'objet de la première partie de l'article. La seconde partie rappellera brièvement les principaux résultats des études sur l'insertion professionnelle des diplômés en France avant de proposer une analyse économétrique de l'insertion des sortants de l'enseignement supérieur en France. Nous mettrons l'accent sur le rôle des diplômes et des filières de formation dans la stabilisation professionnelle des jeunes au cours de leurs premières années de vie active.

\section{Diplômes et insertion des jeunes : perspectives théoriques}

4 Les définitions théoriques de l'insertion considèrent celle-ci comme un processus temporel, qui commence à la sortie du système éducatif lorsque, par exemple, le jeune sortant consacre une part importante de son temps à la recherche d'emploi (Vincens, 1981). Le choix de ce point de départ apparaît cependant contestable dans la mesure où il ne prend pas en compte le passé scolaire ou extra-scolaire des individus. Le jeune peut avoir par exemple repris ses études, après un passage dans le système d'emploi, ou encore avoir exercé des "petits boulots " pour financer ses études... Difficile dans ces cas de considérer la sortie du système éducatif comme le début de la période d'insertion. Vincens (1981) souligne que l'idée d'insertion doit être associée à « l'idée de projet de vie ", la formation initiale est un indicateur de ce qui peut être un projet de vie, mais plus largement l'ensemble des activités de l'individu durant son passage dans le système éducatif peut donner des indications sur son projet de vie, et donc sa période d'insertion.

5 En économie, la théorie du capital humain propose une vision cohérente du parcours scolaire, comme un ensemble de décisions rationnelles qui ont pour principal objectif d'augmenter la productivité future des individus. L'investissement initial a pour but de préparer l'accès à des emplois qui le rentabiliseront (Becker, 1964). Tout au long du 
parcours scolaire, l'individu doit effectuer des arbitrages en matière de formation, à savoir la poursuite (ou non) d'études complémentaires, de stages, ou d'emplois qui pourront accroître sa formation générale ou spécifique. Les individus choisissent rationnellement leur demande d'éducation en fonction des perspectives de débouchés et de revenus futurs sur le marché local du travail ou éventuellement, migrent vers des marchés du travail plus porteurs (Sjaastad, 1962). Dès lors, les choix en matière de formation apparaissent clairement comme un premier acte décisif dans le parcours d'insertion des individus. Les individus les plus formés ont une productivité supérieure sur le marché du travail qui leur permettra d'accéder aux emplois plus qualifiés et mieux rémunérés.

6 L'investissement en formation générale dispensée par le système éducatif, est donc un élément déterminant dans sa trajectoire d'insertion, bien que l'individu puisse tout au long de cette période décider d'investir en formation générale ou spécifique lors de ses activités professionnelles (Keane \& Wolpin, 1997). Au fur et à mesure de leur cursus scolaire ou universitaire, les jeunes peuvent acquérir de nouvelles informations sur leurs chances de succès à l'examen final ou sur leurs opportunités d'emploi sur le marché du travail. Ils peuvent donc en cours d'année modifier leur stratégie d'investissement en capital humain en changeant de filières ou en s'insérant directement sur le marché du travail (Manski, 1989).

7 Il est évident que l'acquisition de capital humain dans le système éducatif n'est pas homogène et va être étroitement liée à la formation dispensée dans chaque filière. Certaines filières donnent une formation très spécialisée et faiblement transférable, ce qui confère aux jeunes un avantage important dans l'accès à un nombre limité d'emplois, mais aucune avantage pour l'autre emplois. En revanche, les différentes filières de formation disposent de nombreuses informations sur les possibilités de carrières et les réseaux d'anciens élèves offrent parfois de bonnes opportunités d'emplois (Curtis \& Warren, 1992).

Le coût direct de l'éducation est d'habitude supporté par les familles et par des subsides publics mais également par un " petit boulot » à temps partiel. L'université de masse permet au jeune de trouver un emploi lors de ses études, tout en bénéficiant d'un statut relativement plaisant et d'un temps libre élevé (Lévy-Garboua, 1976). Or, cette activité peut se révéler un investissement très valorisant dans le cadre de l'insertion professionnelle du jeune (Marsh, 1991 ; Ruhm 1997). De l'étudiant-salarié au salariéétudiant, la frontière est floue et les jeunes sont parfois insérés avant la fin des études (Light, 1998). L'arrêt des études peut simplement refléter une opportunité professionnelle, saisie par le jeune, ou une stabilisation de son activité professionnelle passée. Même si le jeune ne travaille pas durant ses études, Fan (1993) montre que la poursuite d'études correspond d'autant plus à une période de recherche d'emploi que l'incertitude sur les probabilités d'obtenir un emploi rentabilisant la formation est importante. Autrement dit, les inscriptions de précaution dans le système éducatif deviennent plus importantes lorsque le risque associé à l'investissement en capital humain augmente : les jeunes peuvent profiter de la période d'études pour rechercher un emploi tout en ayant la possibilité de prolonger l'investissement initial.

Le niveau d'études peut également influencer la stratégie de recherche d'emploi des jeunes. Ainsi, dans l'estimation de modèles de quête d'emploi, un diplôme élevé est en général corrélé positivement avec l'intensité de recherche (Wadsworth, 1990; Moreau \& Visser, 1991). Cependant aucune explication théorique ne peut a priori établir sans 
ambiguïté l'impact du niveau d'éducation sur les salaires, surtout pour de proches niveaux d'éducation. Le passage par l'enseignement supérieur peut inciter les jeunes à accroître leur salaire de réserve et à refuser certains emplois que des non diplômés peuvent accepter, augmentant alors leur durée d'accès à l'emploi. En revanche, plus instruits et plus âgés, on peut penser qu'ils peuvent mieux se débrouiller dans la recherche de l'emploi. Ainsi, Kettunem (1997) souligne-t-il cette ambiguïté dans l'accès à l'emploi des plus diplômés. Comparer les performances d'insertion de deux niveaux de formation différents est donc un exercice difficile dans la mesure où les jeunes s'adressent à des demandes différentes et n'ont pas la même "utilité de réservation» ${ }^{1}$. Il faut notamment tenir compte du type d'emploi recherché par les jeunes.

La poursuite d'études, l'abandon, l'échec ou la réussite peuvent également s'interpréter en termes d'information imparfaite sur la productivité des jeunes, que l'employeur peut utiliser lors du recrutement. Pour la théorie du filtre, formalisée par Arrow (1973), l'employeur n'achète pas des heures de travail de productivité connue mais sélectionne à l'embauche parmi des personnes dont la productivité potentielle peut être signalée a priori. Cette information est en partie donnée par le diplôme, qui sert de mesure imparfaite des facultés productives plutôt que de preuves de compétences. Le diplôme a alors une valeur de "signal ", très forte lors de l'accès aux premiers emplois, mais qui va décroître au fur et à mesure que les employeurs observent la productivité réelle de l'individu dans l'entreprise ou disposent d'informations sur la trajectoire professionnelle (Belman \& Heywood, 1997). Dans sa version la plus forte, la théorie laisserait présager que, pour un même niveau d'études, les jeunes en situation d'échec scolaire ou issus de filières peu "réputées " peuvent souffrir d'une discrimination par rapport aux autres. Cependant, les disparités d'insertion peuvent provenir d'autres caractéristiques observées ou non observées dans l'enquête, et notamment d'indices si l'on reprend la terminologie de Spence (1974), qui, dans certains cas, ne peuvent être observés que par l'employeur au cours du recrutement (l'expression du candidat, son élégance, sa tenue physique, sa motivation...). Selon Spence, deux critères permettront alors de différencier les jeunes : les "indices » qui concernent les caractéristiques personnelles, intrinsèques des individus et les « signaux » que l'individu peut modifier, tel le niveau de diplôme ou l'expérience professionnelle. Les employeurs peuvent associer des indices relativement bas à des caractéristiques jugées indésirables. Le choix de jeunes diplômés se justifie pour l'employeur par le fait que ces derniers disposent d'une productivité supérieure aux autres, qui leur a permis d'investir dans les études. L'employeur affecte au diplôme un salaire correspondant à la productivité qu'il croit lui être associée. Dans les filières à numerus clausus, la réputation de la filière vient des critères de sélection lors de l'entrée dans celle-ci, qui attestent d'une productivité potentielle élevée (Pigliaru \& Vanini, 1995). Si l'on se réfère à la théorie de la concurrence pour l'accès aux emplois (Thurow, 1974), l'employeur s'intéresse à " l'aptitude à être formé ». Le problème consiste pour l'employeur à recruter et à former les individus qui vont générer la productivité marginale nécessaire à l'emploi avec l'investissement minimal en coût de formation. Afin de minimiser les coûts, les employeurs trient les individus sur la base de ces coûts de formation. Cela conduit à un classement des individus par coûts de formation croissants et in fine, à une file d'attente. Les travailleurs qui possèdent les caractéristiques indiquant les coûts de formation les plus bas sont les premiers embauchés. Cependant, le placement dans la file d'attente n'a aucune raison d'être identique pour tous les emplois : les caractéristiques exigibles pour un emploi de déménageur sont forcément différentes de 
celles pour un emploi de puéricultrice. La position des individus dans cette file d'attente différera en fonction des emplois qui leur seront proposés, bien que certaines caractéristiques comme le diplôme soient généralement appréciées par l'employeur ${ }^{2}$, notamment pour les plus jeunes.

Il ressort de ce rapide balayage théorique un bilan contrasté du rôle du diplôme sur l'insertion professionnelle des jeunes. En général, le diplôme et la filière de formation affectent directement et/ou indirectement la productivité des jeunes dans certains types d'emplois, ce qui leur confère un avantage relatif pour ces emplois. De ce fait, les jeunes ont des stratégies d'insertion souvent différentes en fonction de leur niveau d'études et des caractéristiques de chaque filière (Fellouzis \& Sembel, 1997). Au vu de la théorie économique, il est cependant difficile de trancher a priori sur les conditions d'insertion de ces jeunes : les plus formés devraient accéder plus facilement à un emploi qualifié, au prix d'un éventuel déclassement. Ils peuvent néanmoins avoir un salaire de réserve plus élevé et rester plus longtemps au chômage. Le choix d'une définition précise de la période d'insertion est de ce fait un enjeu particulièrement sensible si l'on veut évaluer l'insertion d'une cohorte par filière de formation. La théorie économique montre également que si le diplôme et la filière de formation ont une influence sur l'insertion des jeunes, d'autres caractéristiques comme l'expérience professionnelle ou les compétences acquises en dehors des systèmes éducatif et productif (Lee, 1986) viennent brouiller la valeur du diplôme sur le marché du travail ${ }^{3}$.

\section{Le diplôme et l'insertion professionnelle des jeunes en France}

Examinons maintenant les différences d'insertion par diplôme et filière de formation en France. Dès le début des années 70, les enquêtes longitudinales ont souvent servi de base à l'analyse des débouchés professionnels des jeunes sur le marché du travail. Le suivi de cohortes de jeunes a permis des analyses de plus en plus techniques, de la simple analyse statistique aux techniques économétriques les plus poussées. Les premières enquêtes longitudinales en France, ont commencé au milieu des années 60 , lors d'études engagées par l'Institut National d'Études Démographiques et le Service Central des Statistiques de la Conjoncture du Ministère de l'Éducation Nationale (Bidon et al., 1970 ; Girard \& Bastide, 1970), puis au début des années 70, par le C.E.J.E.E. ${ }^{4}$ (Vincens \& Boyer, 1975). Elles se sont généralisées ensuite avec l'exploitation des enquêtes de cheminement en France, lancées par le Céreq ${ }^{5}$ à la fin des années soixantedix : les travaux sont passés de simples comparaisons statistiques des taux de placement pour différentes filières de formation à des travaux de plus en plus ambitieux sur la compréhension du marché du travail des jeunes.

13 Les enquêtes de cheminement, proposées en France par le Céreq, se sont particulièrement attachées à restituer la perspective longitudinale du parcours d'insertion en proposant mois par mois les calendriers des différentes étapes du processus de recherche et/ou d'accès à l'emploi. À partir du résultat de ces enquêtes et d'autres enquêtes plus générales comme l'enquête d'emploi, nous dresserons dans un premier temps un portrait très rapide de la littérature empirique de l'insertion des jeunes en France en fonction du niveau de diplômés. Dans un second temps, nous nous focaliserons sur l'insertion des sortants de l'enseignement supérieur en comparant les performances d'insertion d'une cohorte de 6000 jeunes sortant de formation initiale en 
1994 et enquêtés par le Céreq en 1997. L'utilisation de modèles de durée nous permettra de quantifier l'influence des filières des formations sur la qualité et la vitesse d'insertion des jeunes sortis de ces filières.

\section{Un portrait de l'insertion professionnelle en France selon le niveau de formation}

Dans le cadre des études empiriques sur l'insertion professionnelle des jeunes, de nombreux travaux montrent que, dans l'ensemble, les plus diplômés arrivent à s'insérer plus facilement que leurs camarades moins diplômés. Meron et Minni (1995) constatent, par exemple, que les disparités de taux de chômage par niveaux de diplôme se sont accrues depuis 1975, au profit des jeunes diplômés de l'enseignement supérieur. De même, Dumartin (1995) montre que posséder au moins le bac diminue sensiblement la probabilité d'être au chômage et augmente la probabilité d'un emploi à durée indéterminée. Une note de la D.E.P. (1997) oppose le chômage " persistant » des peu diplômés au chômage " intermittent » des diplômés de l'enseignement supérieur qui relèverait davantage de l'instabilité de l'emploi, que de l'exclusion.

Ce constat reste néanmoins dépendant des indicateurs choisis et de la précision des données utilisées. Ainsi, Moneel et Rose (1995) observent que le lien entre la stabilité des parcours et le niveau de formation n'apparaît " ni massif ni régulier » dans le parcours individuel du jeune. Colletaz et al. (1988), précisent que le principal effet du niveau d'études porte sur la fréquence du chômage, le risque de chômage étant sensiblement plus élevé pour les moins diplômés. Les logiques de filières et de spécialités de formation nuancent néanmoins ces résultats : dans le supérieur, les jeunes issus de filières à vocation professionnelle ou à numerus clausus peuvent par exemple connaître de meilleures performances d'insertion (Karaa \& Plassard, 1996). Cependant, là encore, les résultats ne sont pas clairement établis et les performances d'insertion peuvent s'inverser au gré de la conjoncture (Martinelli et al., 1997; Martinelli \& Vergnies, 1995), bien qu'un « emploi stable et bien rémunéré semble aujourd'hui plus qu'hier synonyme de troisième cycle et ou de grande école » comme le soulignent Martinelii et Vergnies $(1999$, p. 1). Pour les niveaux inférieurs au baccalauréat, la hiérarchie des niveaux peut être remise en cause dans l'explication de l'accès à l'emploi (Balsan et al., 1996) : un jeune de niveau IV ayant échoué au baccalauréat a ainsi plus de difficultés qu'un jeune de niveau $\mathrm{V}$ titulaire du B.E.P. ou d'un C.A.P. De plus, à diplôme égal, le type de filière a également une forte influence sur l'insertion des jeunes. Les travaux de Bonnal et al. (1998) indiquent par exemple que les apprentis, au niveau du C.A.P. et du bac professionnel s'insèrent en général mieux que les sortants de formation professionnelle, bien que leur avantage semble diminuer au cours du parcours d'insertion (Sollogoub \& Ulrich, 1998).

Les premiers résultats de l'enquête "Génération 92 ", qui présente l'intérêt de comparer une génération de sortant de tous les niveaux de formation, indiquent même que cinq ans après la fin des études, la situation des plus formés est " aux antipodes " de celle des sortants aux plus bas niveaux de formation (Martinelli et al., 1999). Que les jeunes soient diplômés ou non et indépendamment des indicateurs utilisés, il existe globalement une hiérarchie incontestable des niveaux de formation (cf. tableau 1). 
TABLEAU I. Insertion de la « génération 1992 »: taux de chômage et salaire médian, cinq ans après la sortie

\begin{tabular}{|l|l|l|l|l|}
\hline \multirow{2}{*}{} & \multicolumn{2}{|l|}{ TAUX DE CHÔMAGE EN MARS 97 } & \multicolumn{2}{l|}{ SALAIRE MÉDIAN EN 1997 (EN F) } \\
\cline { 2 - 5 } & DIPLÔMÉS & NON DIPLÔMÉS & DIPLÔMÉS & NON DIPLÔMÉS \\
\hline C.A.P.-B.E.P. & $15 \%$ & $24 \%$ & 5958 & 5500 \\
\hline BAC & $11 \%$ & $16 \%$ & 6500 & 5708 \\
\hline D.U.T.-B.T.S. & $7 \%$ & - & 7958 & 6175 \\
\hline T cycle universitaire & $5 \%$ & - & 9300 & - \\
\hline $3 `$ cycle universitaire & $7 \%$ & - & 11000 & \\
\hline École d'ingénieurs & $4 \%$ & - & 13867 & \\
\hline École de commerce & $5 \%$ & & 13000 & - \\
\hline
\end{tabular}

17 Source : Céreq (Martinelli et al., 1999).

TABLE 1. The transition for school to work for the school leavers in 1992: unemployment rate and rnedian wages, five years later

Cependant, cet avantage accordé au diplôme pour l'insertion n'est que relatif. En effet, de nombreux résultats empiriques laissent penser que les différences d'insertion constatées entre les niveaux d'études ne sont pas dues au dynamisme d'un marché du travail des diplômés, mais plutôt à des mécanismes croissants de déclassement ${ }^{6}$ des jeunes diplômés qui viennent concurrencer leurs camarades moins diplômés. Les travaux de Minni et Poulet (1999b), Forgeot et Gautier (1997), Béduwé et Espinasse (1995), Kirsh et Desgoutte (1996), Baudelot et Glaude (1989), mettent tous en évidence un déclassement des diplômés, au niveau salarial, comme au niveau des emplois, dont la conséquence majeure est un effet de report du chômage sur les moins diplômés (Gautier, 1994). S'ils ne trouvent pas d'emploi correspondant à leur niveau de capital humain, lesjeunes peuvent concurrencer les moins diplômés et accepter des emplois ne correspondant pas à leurs attentes initiales (niveau de salaire, position dans la hiérarchie, ...). Un simple regard sur l'évolution du niveau de diplôme et l'évolution des emplois suffit à montrer que ces déclassements sont " presque » automatiques, avec la hausse générale de l'offre d'éducation (Béduwé \& Espinasse, 1995). L'observation des changement dans la structure des catégories socioprofessionnelles entre 1990 et 1997 révèle l'importance croissante de ces déclassements chez les jeunes pour les plus hauts niveaux de formation ${ }^{7}$. Pour les diplômés de l'Université, il s'est traduit par une augmentation sensible de la part des ouvriers et employés qui est passée de $11 \%$ à $18 \%$ de la population active occupée. Pour les diplômés des Grandes Écoles, il s'amorcent par un léger accroissement des professions intermédiaires.

L'observation de trajectoires d'insertion opposées, même pour des jeunes de niveau de formation sensiblement identique (Béduwé \& Cahuzac, 1997), révèle l'insuffisance d'une explication unique de l'insertion. Si le diplôme a été jusqu'à présent la clé d'une " bonne » insertion, il n'apparaît plus actuellement comme la garantie d'un passeport 
automatique pour l'emploi. Bien qu'il permette dans la majorité des cas de se situer en haut de la " file d'attente " et même parfois de l'éviter, il n'est pas une condition suffisante pour un accès rapide et définitif à un emploi qualifié. Le diplôme devient alors une simple caractéristique individuelle parmi un ensemble de caractéristiques repérables par l'employeur.

\section{L'entrée dans la vie active des diplômés de l'enseignement supérieur sortis en 1994: une analyse économétrique de la durée d'insertion}

Regardons de manière plus précise l'insertion des jeunes sortants de l'enseignement supérieur en France. A un certain niveau de généralité, les diplômés de l'enseignement s'insèrent mieux que les autres et accèdent à des emplois plus qualifiés, mais cette proposition recouvre des réalités très différentes selon les filières de formation (Degennes et ai., 1999). Nous nous focaliserons plus spécialement sur la durée de la période d'insertion. Afin d'essayer de mieux saisir l'influence respective de chaque filière, nous utiliserons dans ce paragraphe une enquête rétrospective du Céreq sur les diplômés de l'enseignement supérieur sortis en 1994. Cette enquête concerne les diplômés d'instituts universitaires de technologie, de sections de techniciens supérieurs, d'écoles d'ingénieurs et de commerce, de deuxième et de troisième cycles universitaires (hors santé).

Il est possible de définir, à l'instar de Vernières (1997), la fin de la période d'insertion comme l'accès à une position stabilisée dans le système d'emploi. Le problème, si l'on veut rendre opérationnelle cette définition, est de préciser d'un point de vue statistique ce que peut être une position stabilisée pour chaque individu. Cela renvoie aux problèmes des définitions subjectives et objectives de l'insertion (Vincens, 1997), c'està-dire basée sur la perception individuelle de chaque jeune ou sur des indicateurs statistiques commun à tous. Dans le cas de définition objective, cela pose également le problème de trouver des critères pertinents, adaptés à chaque cohorte et susceptibles de déterminer si les jeunes sont insérées on non (Giret, 1998). Il est évident que pour les jeunes issus de l'enseignement supérieur, la fin de la période d'insertion ne peut être assimilée à l'accès à un premier emploi, parfois considéré comme un emploi d'attente (Bestion, 1981). En effet, on peut penser que les jeunes ayant accédé à un troisième cycle, privilégient des emplois plus qualifiés, mieux rémunérés ou plus stables parce qu'ils ont une « utilité de réservation " plus élevée. Ils préféreront ce type d'emplois à d'autres emplois moins qualifiés, au risque de connaitre dans un premier temps, une recherche d'emploi plus longue.

Nous proposons donc de sélectionner certains indicateurs susceptibles de mieux définir ce que pourrait être l'accès à une position stabilisée pour les jeunes issus de l'enseignement supérieur. En plus de l'accès au premier emploi, nous prenons deux autres critères, basés sur la qualification et la stabilité pour comparer l'insertion de ces jeunes. On notera :

Dl: la durée d'accès au premier emploi,

D2: la durée d'accès au premier emploi, s'il s'agit d'un emploi de cadre, ou sinon, la durée d'accès à une période d'emploi d'au moins six mois,

D3: la durée d'accès à une période d'emploi d'au moins un an. 

correspondant à des paliers « clés » dans l'étude de la trajectoire d'insertion, auraient pu être proposées. Ces durées nous ont cependant été imposées par la nature des données, qui ne nous fournit des informations que sur le premier et le dernier emploi occupé par le jeune. Il nous est donc impossible pour D2 de calculer la durée d'accès au premier emploi de cadre. D2 permet de distinguer ce que l'on pourrait appeler un emploi d'attente ou un emploi "alimentaire ", souvent temporaire et déqualifié, permettant aux jeunes de financer de futures recherches, d'une « position stabilisée ». Cependant, si la situation d'emploi se pérennise dans le temps, le jeune peut accepter son statut et se stabiliser.

Nous ferons par ailleurs l'hypothèse que le début de la période d'insertion correspond à la date de sortie du système éducatif. Cette hypothèse est discutable dans la mesure où une partie des étudiants travaillent durant leurs études. Cependant, comme le remarque Vincens (1997), cette définition de l'état initial de l'insertion reste largement utilisée dans les enquêtes d'insertion en France et garantit pour la majorité des formations, une homogénéité suffisante de la cohorte.

Globalement, près de $95 \%$ des jeunes sortant de l'enseignement supérieur ont accédé à l'emploi au cours de la période d'observation ${ }^{8}$, avec une durée moyenne de 2,3 mois. $9,5 \%$ n'ont jamais obtenu un poste de cadre ou plus de 6 mois d'emploi consécutifs et $20,2 \%$ ne sont parvenus à une période d'au moins un an d'emploi stable. Un rapide examen des tableaux 2 et 3 montre que la dernière filière de formation suivie par les jeunes influence leur performance d'insertion. Les jeunes sortants d'un doctorat, d'un D.E.A. ou D.E.S.S. de sciences, d'une école de commerce ou d'une école d'ingénieur s'insèrent en général mieux que leurs camarades. Cependant, les différences dans les taux d'insertion et dans les durées d'insertion sont relativement faibles. Par ailleurs, il est d'autant plus difficile de savoir si ces effets sont significatifs que seuls sont pris en compte, dans le tableau 3, les jeunes effectivement insérés, ce qui entraîne par conséquent un problème de censure 9 .

TABLEAU 2. Taux d'insertion en fonction de la filière de formation suivie

\begin{tabular}{|c|c|c|c|c|c|c|c|c|c|c|}
\hline is & $\begin{array}{c}\text { EOOIE } \\
\text { DE } \\
\text { COSAERCEE }\end{array}$ & $\begin{array}{c}\text { ÉCOLE } \\
\text { D'INGÉNIEURS }\end{array}$ & $\begin{array}{l}\text { D.E.A. } \\
\text { DE } \\
\text { SCIENCES }\end{array}$ & $\begin{array}{l}\text { D.E.A. } \\
\text { DE SC. } \\
\text { SOCIAL. } \\
\text { ET HUM. }\end{array}$ & $\begin{array}{c}\text { DOCTORAT } \\
\text { DE } \\
\text { SCIENCES }\end{array}$ & $\begin{array}{c}\text { DOCTORAT } \\
\text { DE SCIENCE } \\
\text { SOC. ET HUM. }\end{array}$ & L.U.T. & B.T.S. & $\begin{array}{l}2^{\text {sd }} \text { CYCIE } \\
\text { SCIENCES }\end{array}$ & $\begin{array}{l}z^{2} \text { CYCLE } \\
\text { sCIENCES } \\
\text { SOC. HUM. }\end{array}$ \\
\hline 1 & 97,4 & 95,2 & 98,8 & 95,6 & 96,9 & 93,3 & 96,3 & 96,9 & 95,8 & 89,4 \\
\hline I. & 95,8 & 94,7 & 94,1 & 92,7 & 96,0 & 92,3 & 90,3 & 92,7 & 86,5 & 84,9 \\
\hline (1) & 83,5 & 85,4 & 87,5 & 82,3 & 88,9 & 85,9 & 78,7 & 75,7 & 85,6 & 77,4 \\
\hline
\end{tabular}

TABLE 2. Transition rates in each education area 
TABLEAU 3. Durées moyennes d'insertion (en mois ${ }^{10}$ )

\begin{tabular}{|c|c|c|c|c|c|c|c|c|c|c|}
\hline ate & $\begin{array}{c}\text { ECOLE } \\
\text { DE } \\
\text { COMMERCE }\end{array}$ & $\begin{array}{c}\text { ÉCOLE } \\
\text { D'INGÉNIEURS }\end{array}$ & $\begin{array}{c}\text { D.E.A. } \\
\text { DE } \\
\text { SCIENCES }\end{array}$ & $\begin{array}{l}\text { D.E.A. } \\
\text { DE SC. } \\
\text { SOCIAL. } \\
\text { ET HUM. }\end{array}$ & $\begin{array}{l}\text { DOCTORAT } \\
\text { DE } \\
\text { SCIENCES }\end{array}$ & $\begin{array}{c}\text { DOCTORAT } \\
\text { DE SCIENCE } \\
\text { SOC. ET HUM. }\end{array}$ & I.U.T. & B.TS. & $\mid \begin{array}{l}2^{\text {nd }} \text { CYCLE } \\
\text { SCIENCES }\end{array}$ & $\begin{array}{l}2^{\text {20 }} \text { CYCLE } \\
\text { SCIENCES } \\
\text { SOC. HUM. }\end{array}$ \\
\hline d & 2,2 & 2,5 & 2,1 & 2,3 & 1,3 & 1,6 & 2,6 & 2,2 & 3,1 & 2,4 \\
\hline " & 3,0 & 2,7 & 2,3 & 2,6 & 1,3 & 1,8 & 4,4 & 4,1 & 3,6 & 3,2 \\
\hline ij & 4,1 & 3,4 & 4,2 & 3,4 & 3,2 & 3,6 & 4,8 & 5,1 & 4,5 & 3,8 \\
\hline
\end{tabular}

TABLE 3. Average duratkens of transition

Il convient donc d'examiner les facteurs explicatifs de l'insertion des individus dans un contexte plus global de manière à quantifier et normer les effets des caractéristiques individuelles pour les différentes durées d'insertion. Nous nous proposons d'utiliser l'économétrie des modèles de durée afin de mieux appréhender aspect temporel du processus d'insertion et de tenir compte des problèmes de censure. Nous avons retenu un modèle semi-paramétrique à hasards proportionnels. Cette méthode présente l'avantage de ne pas imposer une loi statistique a priori pour la distribution de la durée. Elle permet d'introduire simultanément plusieurs variables et surtout d'analyser les effets de chaque variable toutes choses égales par ailleurs. En revanche, nous faisons l'hypothèse que les différentes caractéristiques individuelles agissent multiplicativement sur une fonction de risque commune à tous les individus, tout au long du temps. Ainsi, le rapport entre les quotients individuels reste constant au cours du temps. Autrement dit, on suppose ici qu'une variable comme le diplôme aura un effet constant au cours de la période d'observation sur le risque d'insertion ${ }^{11}$. 

Les modèles semi-paramétriques modélisent les

pour un individu ayant les caratis

où $z_{i} \beta=z_{i 1} \beta_{1}+z_{i 2} \beta_{2}+\ldots+z_{i k} \beta_{k}$ et le vecteur colonne $\beta$

des $k$ différentes caractéristiques pour

$$
L=\prod_{i=1}^{n} f\left(t_{i} ; z_{i}\right)^{C_{i}} S\left(t_{i} ; z_{i}\right)^{1-C_{i}}
$$

où $C_{i}=0$ si la durée est censurée, et 1 dans le cas contraire. Cox a alors proposé d'estimer une version semi-paramétrique du modèle où la fonction de hasard de base $h_{0}(t)$ reste non spécifiée, via la maximisation de la vraisemblance partielle (Cox Oakes, 1984, pp. 91-96)

$$
\begin{gathered}
P L=\prod_{i=1}^{n}\left[\frac{\exp \left(\beta x_{i}\right)}{\sum_{j=1}^{n} Y_{i j} \exp \left(\beta x_{j}\right)}\right]^{c_{i}} \\
\text { où } \begin{array}{l}
Y_{i j}=1 s i \quad t_{j} \geq t_{i} \\
\text { et } \quad Y_{i j}=0 s i \quad t_{j}<t_{i}
\end{array}
\end{gathered}
$$

Presntation of the semiparametric model (Cox's method) ${ }^{12}$ diplômés qui accèdent le plus rapidement à l'emploi. Les jeunes sortant après un B.T.S. ont $28 \%$ de chances en plus d'accéder à cet emploi par rapport aux jeunes qui sortent après une inscription en second cycle universitaire de sciences sociales ou humaines. Les jeunes inscrits en doctorats de sciences et dans une moindre mesure, ceux inscrits en D.E.A. ou D.E.S.S. de sciences, tirent également leur épingle du jeu avec des chances d'accès à l'emploi de $27 \%$ et $19,4 \%$ supérieure à celles des universitaires de second cycle en sciences sociales ou humaines. De même, les jeunes inscrits en D.U.T. (+ 17 \%) connaissent une probabilité élevée d'accéder rapidement au premier emploi. Il ne semble pas exister de fortes différences entre les jeunes sortant d'écoles d'ingénieur, d'écoles de commerce ou de troisièmes cycles universitaires de sciences sociales ou humaines. Ces jeunes ont une probabilité d'accéder rapidement à un emploi supérieure de 10 à $15 \%$ par rapport aux universitaires en sciences sociales et humaines. Ces premiers résultats indiquent donc que les jeunes issus de filières de formation courtes et professionnalisées ne sont pas défavorisés dans l'accès au premier emploi. Par rapport aux filières universitaires plus théoriques, le caractère directement opérationnel de cette formation leur permet un accès à l'emploi plus rapide. Il est évident que l'emploi recherché n'est pas le même que pour les autres niveaux de 
formation et que le fait de trouver rapidement un premier emploi n'est pas forcément le signal d'une stabilisation directe. Si l'on se réfère aux deux autres indicateurs de durées retenus pour modéliser la vitesse d'insertion, on constate cette fois une nette hiérarchisation des effets avec le niveau d'études. Les sortants de doctorats, notamment en sciences, les sortants d'écoles d'ingénieurs, et dans une moindre mesure les sortants de D.E.A. et D.E.S.S., accèdent plus rapidement à des emplois stables et plus qualifiés. Ainsi, un jeune sortant d'un doctorat de sciences a $50 \%$ de chances en plus de trouver un emploi de cadre ou un emploi de plus d'un an, par rapport à un universitaire sortant de second cycle. En revanche, aucune différence significative n'existe entre les sortants d'I.U.T., de B.T.S. ou de second cycle universitaire, quelle que soit la discipline.

Ces résultats semblent plaider pour des différences dans les stratégies d'insertion des jeunes sortant de l'enseignement supérieur. On peut penser que les plus diplômés privilégient la qualification et la stabilité de l'emploi. Or, du fait d'une utilité de réservation plus élevée et d'un taux d'arrivée d'offre plus faible des

TABLEAU 4. Rôles des caractéristiques individuelles sur les différentes durées ${ }^{14}$

\begin{tabular}{|c|c|c|c|c|c|c|}
\hline & \multicolumn{2}{|l|}{$\mathrm{Dl}$} & \multicolumn{2}{|l|}{ D2 } & \multicolumn{2}{|l|}{ D3 } \\
\hline & $\begin{array}{l}\text { COEF. } \\
\text { (ÉCART- } \\
\text { TYPE) }\end{array}$ & $\begin{array}{l}\text { VARIATION } \\
\text { DU RISQUE } \\
\text { EN \% }\end{array}$ & $\begin{array}{l}\text { COEF. } \\
\text { (ÉCART- } \\
\text { TYPE) }\end{array}$ & $\begin{array}{l}\text { VARIATION } \\
\text { DU RISQUE } \\
\text { EN \% }\end{array}$ & $\begin{array}{l}\text { COEF. } \\
\text { (ÉCART- } \\
\text { TYPE) }\end{array}$ & $\begin{array}{l}\text { VARIATION } \\
\text { DU RISQUE } \\
\text { EN \% }\end{array}$ \\
\hline $\begin{array}{l}\text { Filière de formation Ref : } 2^{\prime \prime i} \\
\text { cycle univ. en sciences humaines } \\
\text { ou sociales }\end{array}$ & & & & & & \\
\hline École de commerce & $\begin{array}{l}0,096 \\
(0,05)\end{array}$ & $101 \% *$ ' & $\begin{array}{l}0{ }^{\prime}{ }^{\circ} 66 \\
(0,05)\end{array}$ & $.6^{9 \%} \%$ & $\begin{array}{l}0,014 \\
(0,057)\end{array}$ & $1,4 \%$ \\
\hline École d'ingénieur & $\begin{array}{l}0,115 \\
(0,06)\end{array}$ & $12,2 \% *$ । & $\begin{array}{l}0127 \\
(0,067)\end{array}$ & $31 \% * * *$ & $\begin{array}{l}01293 \\
(0,07)\end{array}$ & $341 \% * * * 1$ \\
\hline D.E.A. de sciences exactes & $\begin{array}{l}0,178 \\
(0,10)\end{array}$ & $194 \% *$ & $\begin{array}{l}0,214 \\
(0,10)\end{array}$ & $239 \% * *$ & ${ }^{\circ} 25$ & $253 \% * * 1$ \\
\hline $\begin{array}{l}\text { D.E.A. de sciences sociales et } \\
\text { humaines }\end{array}$ & $\begin{array}{l}0,121 \\
(0,05)\end{array}$ & $129 \% * * 1$ & 01206 & $\begin{array}{l}(0,05) \\
23 \% * * *\end{array}$ & 01213 & $23,8 \% * * * 1$ \\
\hline Doctorat de sciences exactes & $\begin{array}{l}0,238 \\
(0,07)\end{array}$ & $27 \% * * *$ & $\begin{array}{l}01388 \\
(0,07)\end{array}$ & $475 \% * * *$ & 0126 & $297 \% * * * 1$ \\
\hline $\begin{array}{l}\text { Doctorat de sciences } \\
\text { sociales et humaines }\end{array}$ & $\begin{array}{l}01141 \\
(0,05)\end{array}$ & $15,1 \% * * *$ & $\begin{array}{l}01288 \\
(0,056)\end{array}$ & $334 \% * * *$ & 01221 & $248 \% * * * 1$ \\
\hline D.U.T. & $\begin{array}{l}0,159 \\
(0,07)\end{array}$ & & $\begin{array}{l}-0002 \\
(0,07)\end{array}$ & $-0,2 \%$ & $\begin{array}{l}-0,023 \\
(0,08)\end{array}$ & $23 \%$ \\
\hline B.T.S. & $\begin{array}{l}0,252 \\
(0,06)\end{array}$ & $28,7 \% * * *$ & $\begin{array}{l}0,098 \\
\left(0^{\prime}, 06\right)\end{array}$ & $13 \%$ & 0,06 & $6,2 \%$ \\
\hline
\end{tabular}




\begin{tabular}{|c|c|c|c|c|c|c|}
\hline $\begin{array}{l}\text { 2" cycle universitaire en } \\
\text { sciences exactes }\end{array}$ & $\begin{array}{l}0,072 \\
(0,12)\end{array}$ & $7 \% * * *$ & $\begin{array}{l}0,048 \\
(0,13)\end{array}$ & $5 \%$ & $\begin{array}{l}0,086 \\
1 \\
(0,134)\end{array}$ & $9 \%$ \\
\hline $\begin{array}{l}\text { Avoir travaillé durant ses } \\
\text { études }\end{array}$ & $\begin{array}{l}0,387 \\
(0,04)\end{array}$ & 1 & $\begin{array}{l}01391 \\
(0,03)\end{array}$ & $479 \% * * *$ & 01409 & $506 \% * * * 1$ \\
\hline \multicolumn{7}{|l|}{$\begin{array}{l}\text { Sexe } \\
\text { Ref: être un garçon }\end{array}$} \\
\hline Être une fille & $\begin{array}{l}-74 \\
0,0 \\
(0,03)\end{array}$ & $-71 \% * * * 1$ & $\begin{array}{l}-81 \\
0,0 \\
(0,03)\end{array}$ & $-78 \% * * 1$ & $\begin{array}{l}-04 \\
0,0 \\
(0,03)\end{array}$ & $\begin{array}{l}-04 \% \\
1\end{array}$ \\
\hline - 2 log vraisemblance & \multicolumn{2}{|l|}{63087} & \multicolumn{2}{|l|}{61219} & \multicolumn{2}{|l|}{55148,16} \\
\hline Test de Wald & \multicolumn{2}{|c|}{$140,687^{* * *}$} & \multicolumn{2}{|c|}{$193,362^{* * *}$} & \multicolumn{2}{|c|}{$165,884^{* * *}$} \\
\hline
\end{tabular}

${ }^{*}$ significatif au seuil de $1 \%{ }^{* *}$ significatif au seuil de $5 \% .{ }^{* * *}$ significatif au seuil de $1 \%($ ) écarttype.

34 Les coefficients estimés en 1" colonne peuvent être interprétés comme le risque de trouver un emploi sous l'influence de la variable considérée (pour chaque date). La second colonne donne le ratio de ce risque. Par exemple, le fait de sortir sur le marché du travail après une école de commerce a pour conséquence d'augmenter le risque d'accès à une position stabilisée définie par Dl de 10,1\% = [exp(0.96)-1 $]^{*} 100$.

TABLE 4. Estimated coefficients for individual characteristics in the transition durations

emplois qualifiés, leur durée d'accès au premier emploi est sensiblement plus élevée. Inversement, les moins diplômés et notamment les sortants de B.T.S. et I.U.T., recherchent avant tout un premier emploi et acceptent donc des emplois moins qualifiés ou plus précaires. Cela rejoint en partie les travaux de Bouffartigue (1994) qui montrent comment se forment des stratégies d'insertion des jeunes sortant d'I.U.T. ou de B.T.S., en acceptant d'abord un emploi moins qualifié et en adoptant ensuite une stratégie de requalification.

La différence de résultats entre les sortants des écoles de commerce, d'ingénieurs et de troisièmes cycles universitaires (hors doctorat) va dans le même sens que celle observée par Sigot et Vergnies (1998) : l'insertion des jeunes issus d'école d'ingénieurs et dans une moindre mesure, celles des troisièmes cycles universitaires, se fait généralement sur des emplois plus qualifiés par rapport aux sortants des écoles de commerce. Il aurait cependant été intéressant de séparer les sortants des écoles de commerce en fonction de la reconnaissance par l'Etat de l'école comme l'ont fait Martinelli et al. (1997). Ils montrent néanmoins que la reconnaissance par l'État ne garantit pas à ces jeunes une meilleure insertion que les jeunes issus d'un 3e cycle universitaire.

Il faut enfin remarquer que l'exercice d'une activité professionnelle avant la fin des études augmente les chances d'une insertion plus rapide, indépendamment de la durée d'insertion retenue. Il est évidemment que certains jeunes ne changent pas d'emploi à la sortie des études et conservent parfois l'emploi initial durant les premières années 
passées sur le marché du travail. L'effet d'un emploi étudiant sur la durée de recherche du premier emploi est souvent " mécanique » et s'estompe lorsque le jeune a effectivement quitté cet emploi avant de sortir sur le marché du travail (Béduwé et aL, 1999). Cependant si l'on introduit d'autres critères pour définir l'insertion comme le salaire, la qualification ou la stabilité des emplois occupés par le jeune, les travaux antérieurs montrent que son influence est généralement positive (Béduwé \& Cahuzac, 1999 ; Béduwé et al., 1999).

\section{Conclusion} Or, selon le diplôme qu'ils possèdent, les jeunes n'ont pas les mêmes stratégies de recherche, d'investissement en capital humain et n'émettent pas les mêmes signaux sur le marché du travail. De plus, ils ne possèdent pas la même productivité pour les employeurs et n'accèdent pas aux mêmes types d'emploi. Si les plus diplômés s'insèrent en général mieux que les autres, il est difficile de savoir si cela est dû à leur performance en matière de recherche d'emploi, à leur productivité supérieure en moyenne quelle que soit le type d'emploi ou aux segments «plus stables » du marché du travail auxquels ils s'adressent. L'analyse de l'insertion des jeunes sortants de l'enseignement supérieur a montré que la forte hétérogénéité des parcours d'insertion dépend au moins partiellement de leur formation initiale. En général, le diplôme et la filière de formation se sont avérés des facteurs déterminants dans l'explication des disparités d'insertion. Leur effet peut cependant varier selon la définition retenue pour caractériser la fin de la période d'insertion.

41 Pour les jeunes sortants de l'enseignement supérieur, les diplômés de filières scientifiques, notamment d'école d'ingénieur et de troisièmes cycles universitaires, tirent leur épingle du jeu en accédant plus rapidement à des emplois plus qualifiés et plus stables. Pour ce type d'emplois, même si les jeunes ont échoué à l'examen final,

L'orientation scolaire et professionnelle, 29/2 | 2000 
l'accès à ces filières donne un avantage relatif, qui leur permet d'accéder rapidement en haut de la « file d'attente » et même parfois de l'éviter.

\section{BIBLIOGRAPHIE}

Arrow, K.J. (1973). Higher Education as a filter. Journal of Public Économies, 2, 193-216.

Balsan, D., Hanchane, S., \& Werquin, P. (1996). Mobilité professionnelle initiale; éducation et expérience sur le marché du travail. Économie et Statistique, 299, 91-106.

Bauclelot, C., \& Glaude, M. (1989). Les diplômes se dévaluent-ils en se multipliant. Économie et Statistique, 225, 3-15.

Becker, G.S. (1964). Human Capital. New York: Columbia University Press.

Béduwé, C., \& Cahuzac, E. (1997). Première expérience professionnelle avant le diplôme, Formation-Emploi, 58, 89-109.

Béduwé, C., Cahuzac, E., \& Giret, J.F. (1999, juin). Travail étudiant et insertion professionnelle des jeunes. Communication présentée aux Journées du Longitudinal, Clermont (parue également en note LI.R.H.E. $\mathrm{n}^{\circ}$ 304).

Béduwé, C., \& Espinasse, J.M. (1995). France : Politique éducative, amélioration des compétences et absorption des diplômés par l'économie. Sociologie du Travail, 4, 27-556.

Béduwé, C., \& Espinasse, J.M. (1996). Concurrences entre générations et accès à l'emploi des jeunes. Formation-Emploi, 55, 19-41.

Belman, D., \& Heywood, J.S. (1997). Sheepskin effects by cohort : implications of job matching in a signaling model. Oxford Economic Papers, 49, 623-637.

Bestion, F. (1981). L'emploi d'attente. Un outil d'analyse de l'insertion professionnelle. Thèse de doctorat de Sciences Économiques, Université Toulouse 1.

Bidou, D., Gontier, G., \& Vrai, P. (1970). Carrières universitaires et perspectives professionnelles. Population, numéro spécial, 137-178.

Bonnal, L., Mendes, S., \& Sofer, C. (1998). Acces to the first job : a comparison between apprenticeship and vocational in France. Document de Recherche LE.O.-C.R.E.S.E.P., d 20. Bouffanigue, P. (1994). La socialisation professionnelle des jeunes B.T.S.-D.U.T., entre diplôme et statut, des identités incertaines. Formation-Emploi, 45, 3-23.

Colletaz, G., Riboud, M., Sofer, C., \& Sollogoub, M. (1988). Analyse économétrique du marché du travail des Jeunes, Rapport du CR.E.S.E.P., Université d'Orléans.

Cox, D.R., \& Oakes, D. (1984). Analysis of Survival Data. Londres : Chapman et Hall.

Curtis, S.J., \& Warner, J.T. (1992). Matchmaker, matchmaker : the effect of old boy networks on job match quality, eamings, and tenure. Journal of Labor Economics, 10, 3, 306-329.

Degenne, A., Stoeffler-Kern, F., \& Werquin, P. (1999). Cheminements de formation dans l'enseignement supérieur et parcours d'insertion professionnelle. Documents Séminaires de Céreq, 141, 1-343. 
Dumartin, S. (1995). Mobilité géographique et insertion professionnelle des jeunes. Économie et Statistique, 283-284, 97-110.

Fang, C. (1993). Schooling as a job process. Economics Letters, 41, 85-91.

Felouzis, G., \& Sembel, N. (1997). La construction des projets à l'université : les quatre cas des filières de masse, Formation-Emploi, 58, 45-60.

Forgeot, G., \& Gautié, J. (1997). Insertion professionnelle des jeunes et processus de déclassement, Économie et Statistique, 304-305, 53-74.

Gautié, J. (1994). Le chômage des jeunes en France, problème de formation ou de file d'attente? Série Documents de Travail de l'LN.S.E.E., Département des Études Économiques d'Ensemble et Département de la Conjoncture.

Girard, D., \& Bastide, H. (1970). La formation et le devenir des élèves de l'agglomération parisienne ayant cessé leurs études entre 1963 et 1969. Population.

Giret, J.F. (1998). Pour une analyse économique de l'insertion professionnelle des jeunes. Thèse pour le Doctorat de Sciences Économiques, Université Toulouse 1.

Iribarne (D'), A. (1986). L'insertion des jeunes dans la vie active, des problématiques de recherche qui restent à construire. In L. Tanguy (Éd.), L'introuvable relation formation-emploi : un état des recherches en France (pp. 89-95). Paris : La Documentation Française.

Keane, M., \& Wolpin, K.I. (1997). The career decisions of young men. Journal of Political Economy, 105, 3, 473-522.

Kettunem J. (1997). Education and unemployment duration. Economics of Education Review, 6, 2, 163-170.

Kirsh, J.L., \& Desgoutte, J.P. (1996). Diplôme et déclassement. C.E.R.E.Q. B.R.E.F., 117, 1-4.

Lee, K.H. (1986). Affective, cognitive, and vocational skills : the employers' perpective. Economies of Education Review, 5, 4, 395-401.

Light, A. (1998). Estimating the retum to schooling : when does the career begin ? Economics of Education Review, 17, 1, 31-45.

Mansuy, M., \& Méhaut, P. (1999). Les enquêtes d'insertion : usages savants, usages décisionnels. In J.J. Paul (Éd.), Administrer, Gérer, Evaluer les Systèmes Éducatifs (pp. 249-272). Paris : E.S.F. Éditeur.

Marchand, M., \& Minni, C. (1997). Premières Informations et Premières Synthèses, Note D.A.R.E.S., 97-12, 52 .

Marsh, H.W. (1991). Employment during high school : character builder or a subversion of academie goals. Sociology of Education, 64, 172-189.

Martinelli, D., \& Vergnies, J.F. (1995). L'insertion professionnelle des diplômés de l'enseignement supérieur se dégrade. C.E.R.E.Q. B.R.E.F., 107, 1-4.

Martinelli, D., \& Vergnies, J.F. (1999). Diplômés de l'enseignement supérieur, la reprise de l'emploi ne profite pas à tous les filières. C.E.R.E.Q. B.R.E.F., 156, 1-4.

Martinelli D., Sigot J.C., \& Vergnies, J.F. (1997). Diplômés de l'enseignement supérieur, l'insertion professionnelle se stabilise mais les écarts s'accentuent. C.E.R.E.Q. B.R.E.F., 134, 1-4.

Martinelli, D., Simon-Zarea, G., \& Werquin, P. (1999). Génération 92, profil, parcours et emploi en 1992. C.E.R.E.Q. B.R.E.F., 149, 1-8. 
Meron, M., \& Minni, C. (1995). Des études à : plus tard et plus difficilement qu'il y a 20 ans. Économie et Statistique, 283-284, 9-32.

Minni, C., \& Vergnies, J.F. (1994). La diversité des facteurs de l'insertion professionnelle. Économie et Statistique, 277-278, 45-61.

Minni, C., \& Poulet, P. (1999)a. L'évolution de la scolarité et de l'insertion professionnelle des jeunes (1996-1998), 1 les sortants du système éducatif. Note d'information de la D.P.D., 99.07, 1-5.

Minni, C., \& Poulet, P. (1999)b. L'évolution de la scolarité et de l'insertion professionnelle des jeunes (1996-1998), 2 les emplois offerts. Note d'information de la D.P.D., 99.08, 1-5.

Moncel, N., \& Rosé, J. (1996). Spécificités et déterminants de l'emploi des jeunes de 18 à 25 ans et de 26 à 29 ans : vers la fin de la transition professionnelle ? Économie et Statistique, 283-284, 53-56.

Moreau, A., \& Visser, M. (1991). Durée du chômage des jeunes en France. Annales d'Économie et Statistique, 20121, 257-278.

Pigliaru, F., \& Vannini, M. (1995). Some consequence of low quality schooling under asymmetric information. Labour, 9, 1, 133-150.

Ruhm, C.J. (1997). Is high school employment consumption or investment? Journal of Labor Economics, 15, 735-776.

Sigot, J.C., \& Vergnies, J.F. (1998). L'insertion professionnelle des diplômés de l'enseignement supérieur. Enquête auprès des sortants de 1994. Documents du C.E.R.E.Q., Série Observatoire, $n^{\circ} 137$. Sjaastad, L. (1962). The costs and returns in human migration, Journal of Political Economy, 70, 80-93.

Sollogoub, M., \& Ulrich, V. (1998, janvier). Apprenticeship versus vocational school : selection bias and transition from school to work. Communication présentée au S.T.T. meeting, Malaga.

Spence, M. (1974). Market Signaling Informational Transfer in Hiring and Related Screening Processes. Harvard University Press.

Stoeffler-Kern, F., \& Tchibozo, G. (1999). Signaux non standards et insertion professionnelle. In Degenne, Lecoutre, Lièvre, \& Werquin (Éds), Insertion, transition professionnelle et identification de processus (pp. 351-360), Documents Séminaires du C.E.R.E.Q.1 142.

Thurow, L. (1974). Measuring the economic benefits of education. In M.S. Gordon (Ed.), Higher Education in the Labor Markets (pp. 374-418). N.Y. : Mac Grow Hill.

Vincens, J. (1981, février). L'insertion dans la vie active. Acte de colloque organisé par l'Institut des Sciences du Travail à Louvain-La-Neuve. Louvain : Institut de Science du Travail.

Vincens, J. (1997). L'insertion professionnelle des jeunes : délimiter un champ de recherche. Formation Emploi, 60, 21-36.

Vincens, J. (1998). L'insertion professionnelle des jeunes : quelques réflexions théoriques. Formation-Emploi, 61, 59-72.

Vincens, J. (1999). Les enquêtes d'insertion, quels miroirs pour quelles images ?. Note LLR.H.E., 298.

Vincens, J., \& Boyer, R. (1975). L'entrée dans la vie active des licenciés de 1970. Études sur le Marché du Travail. Annales de l'Université des Sciences Sociales, 355-407.

Wadsworth, J. (1990). Unemployment benefits and search effort in the U.K. effort, Economica, 17-34. 


\section{NOTES}

1. L'utilité de réservation concerne l'ensemble des caractéristiques minimales de l'emploi « espéré » par l'individu au cours de sa recherche (la stabilité, la qualification, la pénibilité, le salaire...). Le jeune n'accepte pas l'emploi qui n'a pas ces caractéristiques minimales. Cependant, l'utilité de réservation peut baisser lorsque le jeune s'aperçoit que ses exigences ne correspondent pas aux emplois qui lui sont proposés sur le marché du travail.

2. Ainsi Thurow (1975, P. 91) précise : « There is an average ranking for each background class, but ibis ranking is a composite of the ranking of each individual employers ".

3. On peut également se référer à Stoeffler Kern et Tchibozo (1999) pour une réflexion sur le rôle des signaux « non standards » sur l'insertion des jeunes.

4. Centre d'Études Juridiques et Économiques de l'Emploi.

5. Centre d'Études et de Recherches sur les Qualifications.

6. Forgeot et Gautier (1997, p. 53) définissent le déclassement par le fait de posséder un niveau de formation a priori supérieur à celui requis pour l'emploi occupé.

7. Source : Enquête Emploi,jeunes de 30 ans et moins.

8. Ces jeunes sont suivis sur une trentaine de mois après leur sortie du système éducatif. Cependant, une partie des garçons a effectué le service militaire et n'a pu être observée dans la vie active sur l'ensemble de la période. Afin de comparer l'insertion des sortants sur une durée plus ou moins identique, nous n'avons retenu qu'une population de 4293 jeunes pour lesquels nous disposions de toutes les informations sur leur trajectoire individuelle au cours d'une période d'au moins 19 mois dans la vie active. Cela a permis de limiter le biais lié à la durée du service militaire.

9. Une des principales difficultés dans l'étude de l'insertion est due à la présence de censure, c'est à de dire de trous dans l'information qui existent quand une partie de la trajectoire individuelle manque. Lorsque l'on prend comme point de départ de la période d'insertion la sortie du système éducatif, le problème de la censure (ou troncature) à gauche ne se pose pas puisque dans ce cas, le début de l'enquête correspond généralement au début de la période que l'on souhaite observer. En revanche, on peut penser que tous les individus ne s'inséreront pas avant la fin de l'enquête, surtout si la durée de l'enquête est courte. Il est donc impossible de connaître pour ces derniers leur durée exacte d'insertion. Il s'agit alors d'un problème de censure (ou troncature) à droite.

10. Nous ne retenons ici que les jeunes qui sont insérés pour chaque définition.

11. Globalement, cette hypothèse n'a pas été invalidée par une première analyse non paramétrique.

12. La vraisemblance correspond à la probabilité d'observer des données effectivement recueillies. La fonction de séjour étant continue, une observation d'occurrence à la date $t$ contribue à la vraisemblance par $f(t$,$) , la densité de$ probabilité d'occurrence en $t$. En revanche si l'observation est censurée (ou tronquée) en $t$, sa contribution correspond à la probabilité de séjour au-delà de la date $t_{\text {, }}$, soit $S(t)$.

13. Nous avons également introduit une variable concernant la réussite à l'examen final, l'échec à cet examen pouvant être perçu comme un signal pénalisant par l'employeur. Les coefficients associés à cette variable n'ont jamais été significatifs. Nous ne présentons pas dans le texte les résultats de ces estimations.

14. L'individu de référence est un jeune homme, qui a quitté l'enseignement supérieur après un second cycle universitaire en sciences sociales ou humaines et qui n'a pas travaillé durant ses études.

L'orientation scolaire et professionnelle, 29/2 | 2000 


\section{RÉSUMÉS}

Cet article étudie le rôle du diplôme dans les premières années de vie active des jeunes. Se référant à l'économie du travail et de l'éducation, la première partie aborde la question des relations entre diplôme, filière de formation et insertion professionnelle des jeunes. La seconde partie présente ensuite les principaux résultats des travaux sur l'insertion professionnelle des diplômés en France, à partir d'une revue de la littérature et d'estimations économétriques sur l'insertion des diplômés de l'enseignement supérieur. Les résultats montrent que l'influence du diplôme et de la filière de formation sur le parcours d'insertion est souvent très complexe bien que les plus diplômés accèdent en général à des emplois plus stables et plus qualifiés.

The aim of the study was to investigate whether different educational qualifications are likely to lead to diffèrent transition patterns. The first part of the article develops several theoretical hypotheses regarding the specific way in which the educational qualifications of school leavers influence their school $\mathrm{w}$ work transition. The second part reviews some empirical evidence of differences in transition related to qualifications in France. Using longitudinal data from the C.E.R.E.Q., we focused on the transition of school leavers of higher education in 1994. Results provide evidence that qualifications have complex effects on the transition process and affect the employment probability only for permanent and qualified jobs.

\section{INDEX}

Mots-clés : Insertion professionnelle, diplôme, enquête longitudinale, modèles de durée, enseignement supérieur

Keywords : Transition from school to work, qualification, longitudinal data, duration models, higher education

\section{AUTEUR}

\section{JEAN-FRANÇOIS GIRET}

est économiste et chargé d'études au Département des Entrées dans la Vie Active du C.E.R.E.Q. (1). Il travaille sur l'insertion professionnelle des jeunes, notamment à partir de l'exploitation des enquêtes longitudinales du C.E.R.E.Q. Au moment de la rédaction de cet article, il était chargé d'études au L.I.R.H.E. et est toujours chercheur associé dans ce laboratoire. 